\title{
SYNTHESIS AND HPLC ENANTIOSEPARATION OF DERIVATIVES OF THE 3-HYDROXYPHENYLETHANONE
}

\author{
${ }^{1}$ Čižmáriková, R. $-{ }^{1}$ Némethy, A. $-{ }^{1}$ Valentová, J. $-{ }^{2}$ Hroboňová, K. - \\ ${ }^{i}$ Bruchatá, $K$. \\ ${ }^{1}$ Comenius University in Bratislava, Faculty of Pharmacy, \\ Department of Chemical Theory of Drugs, \\ ${ }^{2}$ Slovak University of Technology in Bratislava, Faculty of Chemical and \\ Food technology, Institute of Analytical Chemistry
}

\begin{abstract}
Within the framework of the study of the synthesis and high-performance liquid chromatography (HPLC) enantioseparation the series of 9 derivatives of 3-hydroxyphenylethanone was prepared by a well-tried method. The structure of the prepared compounds was confirmed on the basis of interpretation of the IR, UV, ${ }^{1} \mathrm{H}$ NMR and ${ }^{13} \mathrm{C}$ NMR spectra. An enantioseparation of prepared compounds was performed using HPLC on a native teicoplanin (Chirobiotic T) and the amylose tris (3,5-dimethylphenylcarbamate) (Chiralpak AD) chiral stationary phases, which is more suitable for the enantioseparation of all prepared compounds especially with heterocycles in the basic part of a molecule.
\end{abstract}

Keywords: aryloxyaminopropanol - hydroxyphenylethanone - enantioseparation HPLC - Chirobiotic T-Chiralpak AD

\section{INTRODUCTION}

Aryloxyaminopropanol type compounds possess in their structure a single stereogenic centre and exist as stereoisomers. Their racemic compounds can be resolved to enantiomers by means of several analytical methods such as HPLC (Matchett et al., 1996, Park et al., 2000, Makamba et al. 1998; Haginaka et al., 1999, Henriksson et al., 1999, Sharma et al., 1995), GC (Gyllenhaal et al., 1985, Donnecke et al., 1996, Abe et al., 1995, Juvancz et al., 1993), TLC (Bhushan \& Arora, 2003; Bhushan \& Tanwar, 2008, Čižmáriková et al., 2010) or CE (Zhang et al., 2008, Beck \& Neau, 2000; Proksa, 1999; Proksa \& Čižmáriková, 2001). The most widely technique used for separation of the enantiomers have been HPLC on different chiral stationary phases (CSP) such as 
$\beta$-cyclodextrin (Matchett et al., 1996; Park et al., 2000), immobilized proteins (Makamba et al., 1998, Haginaka et al., 1999, Henriksson et al., 1999), Pirkle-type phases (Petersen et al., 1997), and cellulose and amylose-based phases (Aboul-Enein \& Bakr, 1998; Valentova et al., 2003). In our previous studies (Čižmáriková et al., 2003; Hroboňová et al., 2004, 2005) the enantioseparation of the racemic aryloxyaminopropanol type compounds was studied. In these papers HPLC techniques with chiral stationary phases based on macrocyclic antibiotics (vancomycin, teicoplanin, teicoplanin aglycone, permethylated teicoplanin aglycone), cyclodextrins- $\beta$ and $\gamma$ and amylose tris (3,5-dimethylphenylcarbamate) were used.

A common mobile phase that consisted of methanol/acetonitrile/acetic acid/triethylamine (45/55/0.3/0.2 v/v/v/v) (Bruchatá et al., 2010; Čižmáriková et al., 2003) was used in all cases of the enantioseparation using macrocyclic antibiotics. Comparing the separation on a teicoplanin column containing carbohydrate moieties (Chirobiotic T), a teicoplanin column without carbohydrate moieties (Chirobiotic TAG) and a methylated teicoplanin column without carbohydrate moieties (Chirobiotic TAGmethylated), the retention factors were increased in the order: $\mathrm{T}<\mathrm{TAG}<\mathrm{TAG}-$ methylated.

Poor separation of enantiomers was obtained on $\beta$ and $\gamma$-cyclodextrins chiral stationary phases.

Enantioseparation using Chiralpak AD CSP with mobile phase hexane/ethanol/methanol/diethylamine was investigated at the novel series of aryloxyaminopropanol type compounds. The influence of mobile phase composition, particularly alcohol modifier content and composition, an analyte retention and separation were determined; the final composition being hexane/ethanol/methanol/diethylamine $(85 / 3.75 / 11.25 / 0.1 \quad \mathrm{v} / \mathrm{v} / \mathrm{v} / \mathrm{v})$. The analyte structure, including the position and nature of aromatic substitution, steric bulk of the nitrogen alkyl substituent and length and bulk of the side chain were found to influence both retention and chiral discrimination.

\section{EXPERIMENTAL DETAILS}

The melting points were determined using a Kofler Micro Hot Stage and were quoted uncorrected. The purity of the prepared compounds was assessed using Silica gel plates UV 254 (Merck), and the solvent system of ethylacetate/diethylamine $(9.5 / 0.5 \mathrm{v} / \mathrm{v})$ was used. UV spectra were run on spectrophotometer GENESYS 10s UV-Vis in methanol. Concentration of compounds was about $10^{-4} \mathrm{~mol} . \mathrm{dm}^{-3}$. IR spectra were recorded using Nicolet 6700 (Termo Scientific). ${ }^{1} \mathrm{H}$ NMR and ${ }^{13} \mathrm{C}$ NMR were recorded on the Varian Gemini 2000 Spectrometer operating at $300 \mathrm{MHz}$ for protons.

\section{HPLC-chromatography}

\section{Instruments}

HPLC studies were performed with a Hewlett Packard (series 1 100) HPLC-system consisting of a quaternary pump equipped with an injection valve (Rheodyne) and a diode array detector. The macrocyclic chiral stationary phase was Chirobiotic T (250 
$\times 4 \mathrm{~mm}$ I.D. particle size $5 \mu \mathrm{m}$ ) (Advanced Separation Technologies. Inc. USA). The mobile phase was a mixture of methanol/acetonitrile/acetic acid/triethylamine $(45 / 55 / 0.3 / 0.2 \mathrm{v} / \mathrm{v} / \mathrm{v} / \mathrm{v})$. The separation was carried out at a flow rate of $1 \mathrm{ml} \cdot \mathrm{min}^{-1}$ and column temperature was $23^{\circ} \mathrm{C}$. The chromatograms were scanned at $270 \mathrm{~nm}$. The injection volume was $20 \mu \mathrm{l}$. The analytes were dissolved in methanol (concentration $1 \mathrm{mg} \cdot \mathrm{ml}^{-1}$ ).

The experimental tasks for second studies were carried out using HPLC system AGILENT (series 1200), consisting of a quaternary pump and a diode detector.

HPLC was carried out using the chiral stationary phases (Chiralpak AD) based on the amylose tris (3,5-dimethylphenylcarbamate) (250x4.6 $\mathrm{mm}$ I.D. particle size $5 \mu \mathrm{m})$. The mobile phase consisted of hexane/ethanol/methanol/diethylamine $(85 / 3.75 / 11.25 / 0.1 \mathrm{v} / \mathrm{v} / \mathrm{v} / \mathrm{v})$. The samples for analysis were prepared as approximately $1 \mathrm{mg} . \mathrm{ml}^{-1}$ solution in mobile phase. Separation was carried out at a flow rate of $0.8 \mathrm{ml} . \mathrm{min}^{-1}$, with a column temperature maintained at $25{ }^{\circ} \mathrm{C}$. The chromatograms were scanned at wavelength $267 \mathrm{~nm} \pm 8 \mathrm{~nm}$.

\section{Chromatographic characteristics}

The separation factor was expressed as $\alpha=\mathrm{k}_{1} / \mathrm{k}_{2}$, where $\mathrm{k}_{1}, \mathrm{k}_{2}$ are the retention factors for the first and second eluting enantiomer. The retention factor $\mathrm{k}$ was calculated as follows: $\mathrm{k}_{1}=\left(\mathrm{t}_{1}-\mathrm{t}_{0}\right) / \mathrm{t}_{0}$ and $\mathrm{k}_{2}=\left(\mathrm{t}_{2}-\mathrm{t}_{0}\right) / \mathrm{t}_{0}$, where $\mathrm{t}_{0}$ is the dead elution time and $\mathrm{t}_{1}$ and $\mathrm{t}_{2}$ are the elution times of enantiomers 1 and 2. The stereochemical resolution factor $\left(R_{s}\right)$ of the first and the second eluting enantiomer was calculated as the ratio of the difference between the retention times $t_{1}$ and $t_{2}$ to the sum of the two peaks' widths $\mathrm{w}_{1}$ and $\mathrm{w}_{2}: \mathrm{R}_{\mathrm{S}}=2\left(\mathrm{t}_{2}-\mathrm{t}_{1}\right) /\left(\mathrm{w}_{1}+\mathrm{w}_{2}\right)$.

\section{Chemicals}

All HPLC grade solvents were obtained from Merck (Germany).

\section{Synthesis}

[3-(2-hydroxy-3-alkylaminopropoxy)phenyl]ethanone and

[3-(2-hydroxy-3-heterocyclopropoxy)phenyl]ethanone.

To $0.15 \mathrm{~mol}$ of 3-hydroxyphenylethanone and $11 \mathrm{~g} 85 \% \mathrm{KOH}, 3 \mathrm{~mol}(235 \mathrm{ml})$ of chloromethyloxirane was gradually added. The mixture reacted for $4 \mathrm{~h}$ with stirring at the temperature of $50-55{ }^{\circ} \mathrm{C}$. The produced $\mathrm{KCl}$ was sucked off and residual chloromethyloxirane was distilled off in a vacuum. The residue was extracted with chloroform and the organic layer was shaken with $\mathrm{NaOH}$ solution $\left(2 \mathrm{~mol}^{-1} \mathrm{l}^{-1}\right)$ and saturated $\mathrm{NaCl}$ solution. The chloroform solution was dried over anhydrous $\mathrm{MgSO}_{4}$ and the chloroform was distilled off. Crude epoxide (60\%) was dissolved in ethanol (200 $\mathrm{ml})$ and reacted with respective amine $(20 \mathrm{ml})$. The mixture was kept at $30^{\circ} \mathrm{C}$ for $3 \mathrm{~h}$ and then at a reflux for $4 \mathrm{~h}$. The solvent and the unreacted amine were removed in vacuo, the residue was diluted with $\mathrm{H}_{2} \mathrm{O}(100 \mathrm{ml})$ and the base was taken into diethylether. The extract was dried with $\mathrm{K}_{2} \mathrm{CO}_{3}$. Addition of an ether solution of fumaric or oxalic acid resulted in separation of the salt which was crystallized from an appropriate solvent (ethylacetate or propan-1-ol). Yield and physico-chemical parameters of prepared compounds are listed in Table 1. 
Table 1. Physico-chemical parameters of prepared compounds

\begin{tabular}{|c|c|c|c|c|}
\hline $\begin{array}{l}\text { Compou } \\
\text { nd } \\
\text { Form of } \\
\text { compoun } \\
d\end{array}$ & $\mathrm{R}$ & $\begin{array}{l}\text { Empirical formula } \\
\mathrm{Mr}\end{array}$ & $\begin{array}{l}\text { M.p. }\left[{ }^{\circ} \mathrm{C}\right] \\
\text { Solvent }\end{array}$ & $\begin{array}{l}\text { Yield [\%] } \\
\mathrm{R}_{\mathrm{F}}\end{array}$ \\
\hline $\begin{array}{l}\text { I } \\
\text { Base }\end{array}$ & Isopropylamino & $\begin{array}{l}\mathrm{C}_{14} \mathrm{H}_{21} \mathrm{O}_{3} \mathrm{~N} \\
251.33\end{array}$ & $\begin{array}{l}64-65 \\
\text { Hexane } \\
\end{array}$ & $\begin{array}{l}67 \\
0.25 \\
\end{array}$ \\
\hline $\begin{array}{l}\text { Ia } \\
\text { Fumarate }\end{array}$ & & $\begin{array}{l}\mathrm{C}_{28} \mathrm{H}_{42} \mathrm{O}_{6} \mathrm{~N}_{2} \cdot \mathrm{C}_{4} \mathrm{H}_{4} \mathrm{O}_{4} \\
618.74\end{array}$ & $\begin{array}{l}139-142 \\
\text { ethyl acetate }\end{array}$ & 0.26 \\
\hline $\begin{array}{l}\text { II } \\
\text { Base }\end{array}$ & Tert-butylamino & $\begin{array}{l}\mathrm{C}_{15} \mathrm{H}_{23} \mathrm{O}_{3} \mathrm{~N} \\
265.36\end{array}$ & $\begin{array}{l}73-75 \\
\text { Hexane }\end{array}$ & $\begin{array}{l}70 \\
0.38 \\
\end{array}$ \\
\hline $\begin{array}{l}\text { IIa } \\
\text { Fumarate }\end{array}$ & & $\begin{array}{l}\mathrm{C}_{30} \mathrm{H}_{46} \mathrm{O}_{6} \mathrm{~N}_{2} \cdot \mathrm{C}_{4} \mathrm{H}_{4} \mathrm{O}_{4} \\
646,79\end{array}$ & $\begin{array}{l}147-149 \\
\text { ethyl acetate }\end{array}$ & 0.38 \\
\hline $\begin{array}{l}\text { III } \\
\text { Base }\end{array}$ & Isobutylamino & $\begin{array}{l}\mathrm{C}_{15} \mathrm{H}_{23} \mathrm{O}_{3} \mathrm{~N} \\
265.36\end{array}$ & viscous oil & $\begin{array}{l}79 \\
0.48\end{array}$ \\
\hline $\begin{array}{l}\text { IIIa } \\
\text { Fumarate }\end{array}$ & & $\begin{array}{l}\mathrm{C}_{30} \mathrm{H}_{46} \mathrm{O}_{6} \mathrm{~N}_{2} \cdot \mathrm{C}_{4} \mathrm{H}_{4} \mathrm{O}_{4} \\
646.79\end{array}$ & $\begin{array}{l}112-114 \\
\text { propan-2-ol }\end{array}$ & 0.47 \\
\hline $\begin{array}{l}\text { IV } \\
\text { Base }\end{array}$ & Pyrrolidin-1-yl & $\begin{array}{l}\mathrm{C}_{15} \mathrm{H}_{21} \mathrm{O}_{3} \mathrm{~N} \\
263.34\end{array}$ & viscous oil & $\begin{array}{l}78 \\
0.52 \\
\end{array}$ \\
\hline $\begin{array}{l}\text { Iva } \\
\text { Fumarate }\end{array}$ & & $\begin{array}{l}\mathrm{C}_{30} \mathrm{H}_{42} \mathrm{O}_{6} \mathrm{~N}_{2} \cdot \mathrm{C}_{4} \mathrm{H}_{4} \mathrm{O}_{4} \\
642.75\end{array}$ & $\begin{array}{l}102-105 \\
\text { ethyl acetate }\end{array}$ & 0.53 \\
\hline $\begin{array}{l}\mathrm{IVb} \\
\text { Oxalate }\end{array}$ & & $\begin{array}{l}\mathrm{C}_{30} \mathrm{H}_{42} \mathrm{O}_{6} \mathrm{~N}_{2} \cdot \mathrm{C}_{2} \mathrm{H}_{2} \mathrm{O}_{4} \\
616.71\end{array}$ & $\begin{array}{l}139-141 \\
\text { ethyl acetate }\end{array}$ & 0.51 \\
\hline $\begin{array}{l}\text { V } \\
\text { Base }\end{array}$ & Piperidino & $\begin{array}{l}\mathrm{C}_{16} \mathrm{H}_{23} \mathrm{O}_{3} \mathrm{~N} \\
277.37 \\
\end{array}$ & $\begin{array}{l}84-86 \text { a } \\
\text { Hexane }\end{array}$ & $\begin{array}{l}66 \\
0.66 \\
\end{array}$ \\
\hline $\begin{array}{l}\text { Va } \\
\text { Fumarate }\end{array}$ & & $\begin{array}{l}\mathrm{C}_{32} \mathrm{H}_{46} \mathrm{O}_{6} \mathrm{~N}_{2} \cdot \mathrm{C}_{4} \mathrm{H}_{4} \mathrm{O}_{4} \\
670.79\end{array}$ & $\begin{array}{l}165-7 \\
\text { ethyl acetate }\end{array}$ & 0.67 \\
\hline $\begin{array}{l}\mathrm{VI} \\
\text { Base } \\
\end{array}$ & Azepan-1-yl & $\begin{array}{l}\mathrm{C}_{17} \mathrm{H}_{25} \mathrm{O}_{3} \mathrm{~N} \\
291.39\end{array}$ & $\begin{array}{l}58-60 \\
\text { Hexane }\end{array}$ & $\begin{array}{l}63 \\
0.78\end{array}$ \\
\hline $\begin{array}{l}\text { Via } \\
\text { Fumarate }\end{array}$ & & $\begin{array}{l}\mathrm{C}_{34} \mathrm{H}_{50} \mathrm{O}_{6} \mathrm{~N}_{2} \cdot \mathrm{C}_{4} \mathrm{H}_{4} \mathrm{O}_{4} \\
698.85\end{array}$ & $\begin{array}{l}169-172 \\
\text { ethyl acetate }\end{array}$ & 0.77 \\
\hline $\begin{array}{l}\text { VII } \\
\text { Base }\end{array}$ & Morfolino & $\begin{array}{l}\mathrm{C}_{15} \mathrm{H}_{21} \mathrm{O}_{4} \mathrm{~N} \\
279.34\end{array}$ & $\begin{array}{l}88-89 \mathrm{~b} \\
\text { Hexane }\end{array}$ & $\begin{array}{l}72 \\
0.52\end{array}$ \\
\hline $\begin{array}{l}\text { VIIa } \\
\text { Fumarate }\end{array}$ & & $\begin{array}{l}\mathrm{C}_{30} \mathrm{H}_{38} \mathrm{O}_{8} \mathrm{~N}_{2} \cdot \mathrm{C}_{4} \mathrm{H}_{4} \mathrm{O}_{4} \\
674.75\end{array}$ & $92-93$ & 0.52 \\
\hline
\end{tabular}




\begin{tabular}{|l|l|l|l|l|}
\hline $\begin{array}{l}\text { VIII } \\
\text { Base }\end{array}$ & $\begin{array}{l}\text { 4-Methyl- } \\
\text { piperazine-1-yl }\end{array}$ & $\begin{array}{l}\mathrm{C}_{16} \mathrm{H}_{24} \mathrm{O}_{3} \mathrm{~N}_{2} \\
292.38\end{array}$ & $\begin{array}{l}81-83 \\
\text { Hexane }\end{array}$ & $\begin{array}{l}76 \\
0.71\end{array}$ \\
\hline $\begin{array}{l}\text { VIIIa } \\
\text { Fumarate }\end{array}$ & & $\begin{array}{l}\mathrm{C}_{16} \mathrm{H}_{24} \mathrm{O}_{4} \mathrm{~N}_{2} . \\
2 \mathrm{C}_{4} \mathrm{H}_{4} \mathrm{O}_{4} \\
524.52\end{array}$ & $\begin{array}{l}158-160 \\
\text { ethyl acetate }\end{array}$ & 0.71 \\
\hline $\begin{array}{l}\text { IX } \\
\text { Base }\end{array}$ & $\begin{array}{l}\text { M-(2- } \\
\text { pethoxyphenyl) }\end{array}$ & $\begin{array}{l}\mathrm{C}_{22} \mathrm{H}_{29} \mathrm{O}_{4} \mathrm{~N}_{2} . \\
372.47\end{array}$ & $\begin{array}{l}72-74 \\
\text { Hexane }\end{array}$ & $\begin{array}{l}70 \\
0.77\end{array}$ \\
\hline $\begin{array}{l}\text { IXa } \\
\text { Fumarate }\end{array}$ & & $\begin{array}{l}\mathrm{C}_{22} \mathrm{H}_{29} \mathrm{O}_{4} \mathrm{~N}_{2} \cdot \mathrm{C}_{4} \mathrm{H}_{4} \mathrm{O}_{4} \\
488.54\end{array}$ & $\begin{array}{l}172-173 \\
\text { propan-1-ol }\end{array}$ & 0.78 \\
\hline
\end{tabular}

M.p. melting point, $\mathrm{R}_{\mathrm{F}}$ retardation factor

a) m.p. 86; b) m.p. 91 according to Rastogi et al., (1973).

\section{RESULTS AND DISCUSSION}

The aim of this study was to prepare 9 derivatives of [1-alkylamino- or 1-heterocyclo-2hydroxyphenyl]ethanone with branched alkylamino (isopropyl, terc-butyl and isobutyl) or heterocyclo (pyrolidin-1-yl, piperidino, azepan-1-yl, morpholino, 4-methylpiperazin1-yl and 4-(2-methoxyphenylpiperazin-1-yl) in the basic part of molecule. The compounds were prepared by a two-step synthesis from 3-hydroxyphenylethanone. Oxirane intermediate prepared by the reaction of 3-hydroxyphenylethanone with chloromethyloxirane gives the final products by the reaction with appropriate amines. These were isolated in the form of free bases or salts with fumaric acid and as compounds with oxalic acid (Table 1).

The purity of the final products was checked by TLC using mobile phase ethylacetate/diethylamine $(9.5 / 0.5 \mathrm{v} / \mathrm{v})$. Structures of the prepared compounds were confirmed by IR, UV and NMR spectra (Table 2, 3, 4). The stretching vibrations of the characteristic groups in the IR spectra were $v(\mathrm{OH}) 3139-3295 \mathrm{~cm}^{-1}, v(\mathrm{NH})$ (base) 3073-3074 $\mathrm{cm}^{-1}, v(\mathrm{C}=\mathrm{C}) 1562-1594 \mathrm{~cm}^{-1}$, and $v\left(\mathrm{C}_{\mathrm{Ar}}-\mathrm{O}-\mathrm{C}_{\mathrm{Alk}}\right) 1625-1682 \mathrm{~cm}^{-1}$ (Table 2).

Table 2. Values of the stretching vibration in IR spectra of the prepared bases

\begin{tabular}{|c|c|c|c|c|c|}
\hline Compounds & $\begin{array}{l}\mathbf{v}(\mathrm{OH}) \\
{\left[\mathrm{cm}^{-1}\right]}\end{array}$ & $\begin{array}{l}v(\mathrm{NH}) \\
{\left[\mathrm{cm}^{-1}\right]}\end{array}$ & $\begin{array}{c}\mathrm{v}(\mathrm{C}=\mathrm{C}) \\
{\left[\mathrm{cm}^{-1}\right]}\end{array}$ & $\begin{array}{c}v(C=O) \\
{\left[\mathrm{cm}^{-1}\right]}\end{array}$ & $\begin{array}{c}\mathrm{v}\left(\mathrm{C}_{\mathrm{Ar}}-\mathrm{O}-\mathrm{C}_{\mathrm{Alk}}\right) \\
{\left[\mathrm{cm}^{-1}\right]}\end{array}$ \\
\hline I & 3281 & 3074 & 1594 & 1682 & 1267 \\
\hline II & 3146 & 3073 & 1593 & 1673 & 1219 \\
\hline IIIa & 3139 & & 1562 & $\begin{array}{l}1625 \\
1683 \\
\end{array}$ & 1269 \\
\hline IVb & 3295 & & 1581 & 1680 & 1281 \\
\hline $\mathrm{V}$ & 3154 & & 1592 & 1682 & 1220 \\
\hline VIIa & 3169 & & 1591 & 1678 & 1268 \\
\hline
\end{tabular}


$v$ absorbtion maximum

The UV spectra of bases display bands corresponding to $\pi \rightarrow \pi^{*}$ transition at $\lambda_{\max }=208$ $290 \mathrm{~nm}, \log \varepsilon=2.74-4.07$ (Table 3).

Table 3. Values of $\lambda_{\max }$ and $\log \varepsilon$ in $\mathrm{UV}$ spectra, $[\varepsilon]=\mathrm{m}^{2}$. $\mathrm{mol}^{-1}$

\begin{tabular}{|c|c|c|c|c|c|c|}
\hline Compounds & $\begin{array}{l}\lambda_{\max 1} \\
{[\mathbf{n m}]}\end{array}$ & $\log \varepsilon_{1}$ & $\begin{array}{l}\lambda_{\max } 2 \\
{[\mathbf{n m}]}\end{array}$ & $\log \varepsilon_{2}$ & $\begin{array}{c}\lambda_{\max } 3 \\
{[\mathbf{n m}]}\end{array}$ & $\log \varepsilon_{3}$ \\
\hline $\mathrm{I}$ & 216 & 3.40 & 247 & 3.31 & 305 & 3.36 \\
\hline II & 216 & 3.41 & 248 & 3.34 & 305 & 3.38 \\
\hline IIIa & 215 & 3.83 & 247 & 3.67 & 304 & 3.67 \\
\hline IVa & 215 & 3.72 & 247 & 3.60 & 303 & 3.64 \\
\hline VI & 217 & 3.37 & 248 & 3.27 & 306 & 3.36 \\
\hline $\mathrm{V}$ & 217 & 3.44 & 248 & 3.33 & 306 & 3.39 \\
\hline VIIa & 217 & 3.86 & 249 & 3.72 & 306 & 3.78 \\
\hline IX & 212 & 2.70 & 246 & 3.23 & 284 & 2.69 \\
\hline IXa & 208 & 4.03 & 240 & 3.88 & 280 & 4.07 \\
\hline
\end{tabular}

$\lambda_{\max }$ wave length, $\varepsilon$ molar extinction coefficient

The structure of the aminopropanol chain was proofed by ${ }^{1} \mathrm{H}-\mathrm{NMR}$ and ${ }^{13} \mathrm{C}-\mathrm{NMR}$ spectra (Table 4, Table 5). Two HPLC methods with chiral stationary phases based on native teicoplanin (Chirobiotic T) and derivatised amylose (Chiralpak AD) were used for enantioseparation of racemic compounds in this work.

Table 4. ${ }^{1} \mathrm{H}$ NMR spectral data of bases $\delta$ [ppm] $\left(\mathrm{CDCl}_{3}, \delta, \mathrm{TMS}\right)$

\begin{tabular}{|c|c|}
\hline Compounds & $\delta[\mathrm{ppm}]$ number of protons, multiplicity \\
\hline $\mathbf{I}$ & $\begin{array}{l}1.09\left(\mathrm{~d}, 6 \mathrm{H}, \mathrm{NH}-\mathrm{CH}-\left(\mathrm{CH}_{3}\right)_{2}\right), 2.76(\mathrm{~m}, 1 \mathrm{H}, \mathrm{NH}-\mathrm{CH}), 2.92\left(\mathrm{~m}, 2 \mathrm{H}, \mathrm{Ar}-\mathrm{O}-\mathrm{CH}_{2}\right), \\
2.82(\mathrm{~m}, 1 \mathrm{H}, \underline{\mathrm{CH}}-\mathrm{OH}), 2.46\left(\mathrm{~m}, 2 \mathrm{H}, \mathrm{CH}_{2}-\mathrm{NH}\right), 2.59\left(\mathrm{~s}, 3 \mathrm{H}, \mathrm{CH}_{3}-\mathrm{CO}\right), 7.14(\mathrm{~d}, \\
\left.1 \mathrm{H}, \mathrm{Ar}-\mathrm{H}^{4}\right), 7.26\left(\mathrm{~s}, 1 \mathrm{H}, \mathrm{Ar}-\mathrm{H}^{2}\right), 7.36\left(\mathrm{~d}, 1 \mathrm{H}, \mathrm{Ar}-\mathrm{H}^{6}\right), 7.53\left(\mathrm{t}, 1 \mathrm{H}, \mathrm{Ar}-\mathrm{H}^{5}\right)\end{array}$ \\
\hline Ia & $\begin{array}{l}1.38\left(\mathrm{~d}, 6 \mathrm{H}, \mathrm{NH}-\mathrm{CH}-\left(\mathrm{CH}_{3}\right)_{2}\right), 3.54(\mathrm{~m}, 1 \mathrm{H}, \mathrm{NH}-\mathrm{CH}), 4.19\left(\mathrm{~m}, 2 \mathrm{H}, \mathrm{Ar}-\mathrm{O}-\mathrm{CH}_{2}\right) \\
4.35(\mathrm{~m}, 1 \mathrm{H}, \underline{\mathrm{CH}}-\mathrm{OH}), 3.35\left(\mathrm{~d}, 2 \mathrm{H}, \mathrm{CH}_{2}-\mathrm{NH}\right), 2.65\left(\mathrm{~s}, 3 \mathrm{H}, \mathrm{CH}_{3}-\mathrm{CO}\right), 7.27(\mathrm{~d}, \\
\left.1 \mathrm{H}, \mathrm{Ar}-\mathrm{H}^{4}\right), 7.31\left(\mathrm{~m}, 1 \mathrm{H}, \mathrm{Ar}-\mathrm{H}^{2}\right), 7.63\left(\mathrm{~d}, 1 \mathrm{H}, \mathrm{Ar}-\mathrm{H}^{6}\right), 7.51\left(\mathrm{t}, 1 \mathrm{H}, \mathrm{Ar}-\mathrm{H}^{5}\right), 6.51 \\
\left(\mathrm{~s}, 2 \mathrm{H}, \mathrm{CH}-\mathrm{COO}_{\text {fumar }}\right)\end{array}$ \\
\hline II & $\begin{array}{l}1.13\left(\mathrm{~s}, 9 \mathrm{H}, \mathrm{NH}-\mathrm{C}-\left(\mathrm{CH}_{3}\right)_{3}\right), 4.03\left(\mathrm{~m}, 2 \mathrm{H}, \mathrm{Ar}-\mathrm{O}-\mathrm{CH}_{2}\right), 3.98(\mathrm{~m}, 1 \mathrm{H}, \underline{\mathrm{CH}}-\mathrm{OH}) \\
2.69\left(\mathrm{~d}, 2 \mathrm{H}, \underline{\mathrm{CH}_{2}}-\mathrm{NH}\right), 2.60\left(\mathrm{~s}, 3 \mathrm{H}, \mathrm{CH}_{3}-\mathrm{CO}\right), 7.15\left(\mathrm{~d}, 1 \mathrm{H}, \mathrm{Ar}-\mathrm{H}^{4}\right), 7.26(\mathrm{~s}, 1 \mathrm{H}, \\
\left.\mathrm{Ar}^{2} \mathrm{H}^{2}\right), 7.39\left(\mathrm{~m}, 1 \mathrm{H}, \mathrm{Ar}-\mathrm{H}^{6}\right), 7.55\left(\mathrm{t}, 1 \mathrm{H}, \mathrm{Ar}-\mathrm{H}^{5}\right)+\end{array}$ \\
\hline IIa & $\begin{array}{l}1.43\left(\mathrm{~s}, 9 \mathrm{H}, \mathrm{NH}-\mathrm{C}-\left(\mathrm{CH}_{3}\right)_{3}\right), 4.19\left(\mathrm{~m}, 2 \mathrm{H}, \mathrm{Ar}-\mathrm{O}-\mathrm{CH}_{2}\right), 4.31(\mathrm{~m}, 1 \mathrm{H}, \underline{\mathrm{CH}}-\mathrm{OH}) \\
3.34\left(\mathrm{~d}, 2 \mathrm{H}, \mathrm{CH}_{2}-\mathrm{NH}\right), 2.66\left(\mathrm{~s}, 3 \mathrm{H}, \mathrm{CH}_{3}-\mathrm{CO}\right), 7.31\left(\mathrm{~d}, 1 \mathrm{H}, \mathrm{Ar}-\mathrm{H}^{4}\right), 7.48(\mathrm{~s}, 1 \mathrm{H}, \\
\left.\mathrm{Ar}^{-} \mathrm{H}^{2}\right), 7.51\left(\mathrm{~m}, 1 \mathrm{H}, \mathrm{Ar}-\mathrm{H}^{6}\right), 7.65\left(\mathrm{~m}, 1 \mathrm{H}, \mathrm{Ar}-\mathrm{H}^{5}\right), 6.5\left(\mathrm{~s}, 2 \mathrm{H}, \mathrm{CH}-\mathrm{COO}_{\text {fumar }}\right)\end{array}$ \\
\hline III & $\begin{array}{l}0.92\left(\mathrm{~d}, 6 \mathrm{H}, \mathrm{CH}-\left(\mathrm{CH}_{3}\right)_{2}\right), 1.78\left(\mathrm{~m}, 1 \mathrm{H}, \underline{\mathrm{CH}}-\left(\mathrm{CH}_{3}\right)_{2}\right), 2.89\left(\mathrm{~d}, 2 \mathrm{H}, \mathrm{NH}-\mathrm{CH}_{2}\right), 4.03 \\
\left(\mathrm{~m}, 2 \mathrm{H}, \mathrm{Ar}-\mathrm{O}-\mathrm{CH}_{2}\right), 2.48(\mathrm{~m}, 1 \mathrm{H}, \underline{\mathrm{CH}}-\mathrm{OH}), 3.10\left(\mathrm{~m}, 2 \mathrm{H}, \mathrm{CH}_{2}-\mathrm{NH}\right), 2.58(\mathrm{~s}, 3 \mathrm{H}, \\
\left.\underline{\mathrm{CH}}_{3}-\mathrm{CO}\right), 7.13\left(\mathrm{~d}, 1 \mathrm{H}, \mathrm{Ar}-\mathrm{H}^{4}\right), 7.27\left(\mathrm{~s}, 1 \mathrm{H}, \mathrm{Ar}-\mathrm{H}^{2}\right), 7.35\left(\mathrm{~m}, 1 \mathrm{H}, \mathrm{Ar}-\mathrm{H}^{6}\right), 7.52(\mathrm{t}, \\
\left.1 \mathrm{H}, \mathrm{Ar}-\mathrm{H}^{5}\right)\end{array}$ \\
\hline IIIa & $\begin{array}{l}1.00\left(\mathrm{~m}, 6 \mathrm{H}, \mathrm{CH}-\left(\mathrm{CH}_{3}\right)_{2}\right), 2.11\left(\mathrm{~m}, 1 \mathrm{H}, \underline{\mathrm{CH}}-\left(\mathrm{CH}_{3}\right)_{2}\right), 3.33\left(\mathrm{~m}, 2 \mathrm{H}, \mathrm{NH}-\mathrm{CH}_{2}\right), \\
4.19\left(\mathrm{~m}, 2 \mathrm{H}, \mathrm{Ar}-\mathrm{O}-\mathrm{CH}_{2}\right), 4.39(\mathrm{~m}, 1 \mathrm{H}, \mathrm{CH}-\mathrm{OH}), 2.85\left(\mathrm{~d}, 2 \mathrm{H}, \mathrm{CH}_{2}-\mathrm{NH}\right), 2.65(\mathrm{~s}, \\
\left.3 \mathrm{H}, \underline{\mathrm{CH}}_{3}-\mathrm{CO}\right), 7.27\left(\mathrm{~d}, 1 \mathrm{H}, \mathrm{Ar}-\mathrm{H}^{4}\right), 7.46\left(\mathrm{~s}, 1 \mathrm{H}, \mathrm{Ar}-\mathrm{H}^{2}\right), 7.51\left(\mathrm{~m}, 1 \mathrm{H}, \mathrm{Ar}-\mathrm{H}^{6}\right),\end{array}$ \\
\hline
\end{tabular}




\begin{tabular}{|c|c|}
\hline & $7.67\left(\mathrm{t}, 1 \mathrm{H}, \mathrm{Ar}-\mathrm{H}^{5}\right), 6.55\left(\mathrm{~s}, 2 \mathrm{H}, \mathrm{CH}-\mathrm{COO}_{\text {fumar }}\right)$ \\
\hline IV & $\begin{array}{l}2.08\left(\mathrm{~m}, 4 \mathrm{H}, \mathrm{pyrH}^{2}, 6\right), 1.87\left(\mathrm{~m}, 4 \mathrm{H}, \mathrm{pyrH}^{3,5}\right), 1.83\left(\mathrm{~m}, 2 \mathrm{H}, \mathrm{pyrH}^{4}\right), 4.05(\mathrm{~m}, 2 \mathrm{H}, \\
\left.\mathrm{Ar}-\mathrm{O}-\mathrm{CH}_{2}\right), 3.37(\mathrm{~m}, 1 \mathrm{H}, \mathrm{CH}-\mathrm{OH}), 2.72\left(\mathrm{~m}, 2 \mathrm{H}, \mathrm{CH}_{2}-\mathrm{Npyr}\right), 2.81\left(\mathrm{~m}, 3 \mathrm{H}, \mathrm{CH}_{3}-\right. \\
\mathrm{CO}), 7.12\left(\mathrm{~d}, 1 \mathrm{H}, \mathrm{Ar}-\mathrm{H}^{4}\right), 7.27\left(\mathrm{~s}, 1 \mathrm{H}, \mathrm{Ar}-\mathrm{H}^{2}\right), 7.36\left(\mathrm{~m}, 1 \mathrm{H}, \mathrm{Ar}-\mathrm{H}^{6}\right), 7.53(\mathrm{~m}, \\
\left.1 \mathrm{H}, \mathrm{Ar}-\mathrm{H}^{5}\right)\end{array}$ \\
\hline VI & $\begin{array}{l}2.76\left(\mathrm{~m}, 4 \mathrm{H}, \text { azepH } \mathrm{H}^{2,7}\right), 1.70(\mathrm{~m}, 4 \mathrm{H}, \text { azepH } \\
\left.{ }^{3,6}\right), 1.62(\mathrm{~m}, 4 \mathrm{H}, \text { azepH } \\
\left(\mathrm{m}, 2 \mathrm{H}, \mathrm{Ar}-\mathrm{O}-\mathrm{CH}_{2}\right), 2.56(\mathrm{~m}, 1 \mathrm{H}, \mathrm{CH}-\mathrm{OH}), 2.60\left(\mathrm{~d}, 2 \mathrm{H}, \mathrm{CH}_{2}-\mathrm{Nazep}\right), 2.59(\mathrm{~s}, \\
\left.3 \mathrm{H}, \mathrm{CH}_{3}-\mathrm{CO}\right), 7.50\left(\mathrm{~d}, 1 \mathrm{H}, \mathrm{Ar}-\mathrm{H}^{4}\right), 7.26\left(\mathrm{~s}, 1 \mathrm{H}, \mathrm{Ar}-\mathrm{H}^{2}\right), 7.13\left(\mathrm{~d}, 1 \mathrm{H}, \mathrm{Ar}-\mathrm{H}^{6}\right), \\
7.36\left(\mathrm{t}, 1 \mathrm{H}, \mathrm{Ar}-\mathrm{H}^{5}\right)\end{array}$ \\
\hline VII & $\begin{array}{l}2.49(\mathrm{~m}, 4 \mathrm{H}, \mathrm{morfH} \\
(\mathrm{m}, 6), 3.73(\mathrm{~m}, \mathrm{CH}-\mathrm{OH}), 2.68\left(\mathrm{~m}, 2 \mathrm{H}, \mathrm{CH}_{2}-\mathrm{Nmorf} \mathrm{H}^{3,5}\right), 2.59\left(\mathrm{~m}, 3 \mathrm{~m}, 2 \mathrm{H}, \mathrm{Ar}-\mathrm{O}-\mathrm{CH}_{3}-\mathrm{CO}\right), 7.15(\mathrm{~d}) \\
\left.1 \mathrm{H}, \mathrm{Ar}-\mathrm{H}^{4}\right), 7.27\left(\mathrm{~s}, 1 \mathrm{H}, \mathrm{Ar}-\mathrm{H}^{2}\right), 7.40\left(\mathrm{~m}, 1 \mathrm{H}, \mathrm{Ar}-\mathrm{H}^{6}\right), 7.51\left(\mathrm{~m}, 1 \mathrm{H}, \mathrm{Ar}-\mathrm{H}^{5}\right)\end{array}$ \\
\hline VIII & $\begin{array}{l}2.31\left(\mathrm{~s}, 3 \mathrm{H}, \mathrm{CH}_{3}-\mathrm{N}_{\mathrm{pip}}\right), 2.52\left(\mathrm{~m}, 4 \mathrm{H}, \mathrm{pipH}^{2,6}\right) 2.50\left(\mathrm{~m}, 4 \mathrm{H}, \mathrm{pipH}^{3,5}\right), 4.21(\mathrm{~m}, \\
\left.2 \mathrm{H}, \mathrm{Ar}-\mathrm{O}-\mathrm{CH}_{2}\right), 4.03(\mathrm{~m}, 1 \mathrm{H}, \mathrm{CH}-\mathrm{OH}), 2.64\left(\mathrm{~m}, 2 \mathrm{H}, \mathrm{CH}_{2}-\mathrm{NH}\right), 2.39(\mathrm{~s}, 3 \mathrm{H}, \\
\left.\mathrm{CH}_{3}-\mathrm{CO}\right), 7.15\left(\mathrm{~d}, 1 \mathrm{H}, \mathrm{Ar}-\mathrm{H}^{4}\right), 7.25\left(\mathrm{~s}, 1 \mathrm{H}, \mathrm{Ar}-\mathrm{H}^{2}\right), 7.52\left(\mathrm{~m}, 1 \mathrm{H}, \mathrm{Ar}-\mathrm{H}^{6}\right), 7.39(\mathrm{t}, \\
\left.1 \mathrm{H}, \mathrm{Ar}-\mathrm{H}^{5}\right)\end{array}$ \\
\hline VIIIa & $\begin{array}{l}2.22\left(\mathrm{~s}, 3 \mathrm{H}, \mathrm{CH}_{3}-\mathrm{N}_{\mathrm{pip}}\right), 4.15\left(\mathrm{~m}, 4 \mathrm{H}, \mathrm{pipH}^{2,6}\right) 2.84\left(\mathrm{~m}, 4 \mathrm{H}, \mathrm{pipH}^{3,5}\right), 4.16(\mathrm{~m}, \\
\left.2 \mathrm{H}, \mathrm{Ar}-\mathrm{O}-\mathrm{CH}_{2}\right), 4.28(\mathrm{~m}, 1 \mathrm{H}, \mathrm{CH}-\mathrm{OH}), 3.63\left(\mathrm{~m}, 2 \mathrm{H}, \mathrm{CH}_{2}-\mathrm{Npip}\right), 2.66(\mathrm{~s}, 3 \mathrm{H}, \\
\left.\mathrm{CH}_{3}-\mathrm{CO}\right), 7.27\left(\mathrm{~d}, 1 \mathrm{H}, \mathrm{Ar}-\mathrm{H}^{4}\right), 7.47\left(\mathrm{~s}, 1 \mathrm{H}, \mathrm{Ar}-\mathrm{H}^{2}\right), 7.49\left(\mathrm{t}, 1 \mathrm{H}, \mathrm{Ar}-\mathrm{H}^{6}\right), 7.64(\mathrm{~d}, \\
\left.1 \mathrm{H}, \mathrm{Ar}-\mathrm{H}^{5}\right), 6.55\left(\mathrm{~s}, 2 \mathrm{H}, \mathrm{CH}-\mathrm{COO}_{\text {fumar }}\right)\end{array}$ \\
\hline
\end{tabular}

Table 5. ${ }^{13} \mathrm{C}$ NMR spectral data of bases $\delta[\mathrm{ppm}]\left(\mathrm{CDCl}_{3}, \delta\right.$, TMS)

\begin{tabular}{|c|c|}
\hline Compounds & $\delta$ [ppm] \\
\hline I & $\begin{array}{l}23.13\left(\mathrm{NH}-\mathrm{CH}-\left(\mathrm{CH}_{3}\right)_{2}\right), 26.89\left(\mathrm{CO}-\mathrm{CH}_{3}\right), 49.08\left(\mathrm{NH}-\mathrm{CH}-\left(\mathrm{CH}_{3}\right)_{2}\right), 49.25 \\
\left(\underline{\mathrm{CH}}{ }_{2}-\mathrm{NH}-\right), 68.49(\underline{\mathrm{CH}}-\mathrm{OH}), 70.89\left(\mathrm{ArO}-\mathrm{CH}_{2}-\right), 113,32(\mathrm{ArC} 2), 120.14 \\
(\mathrm{ArC} 4), 121.52(\mathrm{ArC}), 129.74(\mathrm{ArC} 5), 138.59(\mathrm{ArC} 3), 159.05(\mathrm{ArC} 1), 198 \\
(\mathrm{Ar}-\mathrm{CO})\end{array}$ \\
\hline Ia & $\begin{array}{l}20.75\left(\mathrm{NH}-\mathrm{CH}-\left(\mathrm{CH}_{3}\right)_{2}\right), 29.23\left(\mathrm{CO}-\mathrm{CH}_{3}\right), 49.53\left(\mathrm{NH}-\mathrm{CH}-\left(\mathrm{CH}_{3}\right)_{2}\right), 53.94 \\
\left(\mathrm{CH}_{2}-\mathrm{NH}-\right), 68.47\left(\mathrm{ArO}-\underline{\mathrm{CH}}_{2}-\right), 72.45(\underline{\mathrm{CH}}-\mathrm{OH}), 112.64(\mathrm{ArC} 2), 123.45 \\
(\mathrm{ArC} 4), 125.13(\mathrm{ArC5}), 133.03(\mathrm{ArC} 6), 138.17\left(\mathrm{CH}=\mathrm{CH}_{\text {fumar }}\right), 140.72(\mathrm{ArC} 3), \\
160.96(\mathrm{ArC} 1), 177.39\left(\mathrm{COO}_{\text {fumar }}\right), 206.25(\mathrm{Ar}-\mathrm{CO})\end{array}$ \\
\hline II & $\begin{array}{l}26.86\left(\mathrm{CO}-\underline{\mathrm{CH}}{ }_{3}\right), 29.27\left(\mathrm{C}-\left(\underline{\mathrm{CH}}_{3}\right)_{3}\right), 44.66\left(\underline{\mathrm{CH}}_{2}-\mathrm{NH}-\right), 50.52\left(\underline{\mathrm{C}}-\left(\mathrm{CH}_{3}\right)_{3}\right), \\
68.69(\underline{\mathrm{CH}}-\mathrm{OH}), 70.9\left(\mathrm{ArO}-\underline{\mathrm{C}} \mathrm{H}_{2}-\right), 113.34(\mathrm{ArC} 2), 120.12(\mathrm{ArC} 4), 121.44 \\
(\mathrm{ArC} 6), 129.7(\mathrm{ArC} 5), 138.57(\mathrm{ArC} 3), 159.09(\mathrm{ArC} 1), 197.99(\mathrm{Ar}-\mathrm{CO})\end{array}$ \\
\hline IIa & $\begin{array}{l}27.64\left(\mathrm{CO}-\underline{\mathrm{CH}}_{3}\right), 29.25\left(\mathrm{C}-(\underline{\mathrm{CH}})_{3}\right), 46.74\left(\underline{\mathrm{CH}}_{2}-\mathrm{NH}-\right), 60.31\left(\underline{\mathrm{C}}-\left(\mathrm{CH}_{3}\right)_{3}\right), \\
68.76\left(\mathrm{ArO}-\mathrm{CH}_{2}-\right), 72.51(\underline{\mathrm{CH}}-\mathrm{OH}), 116.34(\mathrm{ArC} 2), 123.47(\mathrm{ArC} 4), 125.14 \\
(\mathrm{ArC} 5), 133.04(\mathrm{ArC} 6), 138.17\left(\mathrm{CH}=\mathrm{CH}_{\text {fumar }}\right), 140.76(\mathrm{ArC} 3), 160.98(\mathrm{ArC} 1), \\
177.41\left(\mathrm{COO}_{\text {fumar }}\right), 206.29(\mathrm{Ar}-\mathrm{CO})\end{array}$ \\
\hline III & $\begin{array}{l}20.67\left(\mathrm{CH}-\left(\mathrm{CH}_{3}\right)_{2}\right), 26.85\left(\mathrm{CO}-\mathrm{CH}_{3}\right), 28.36\left(\underline{\mathrm{CH}}-\left(\mathrm{CH}_{3}\right)_{2}\right), 51.82\left(\underline{\mathrm{CH}}_{2}-\mathrm{NH}-\right), \\
57.81\left(\underline{\mathrm{CH}}_{2}-\mathrm{CH}-\left(\mathrm{CH}_{3}\right)_{2}\right), 67.98(\underline{\mathrm{C}} \mathrm{H}-\mathrm{OH}), 70.86\left(\mathrm{ArO}-\underline{\mathrm{CH}}_{2}-\right), 113.33(\mathrm{ArC} 2), \\
120.09(\mathrm{ArC} 4), 121.47(\mathrm{ArC} 6), 129.71(\mathrm{ArC} 5), 138.54(\mathrm{ArC} 3), 159.03 \\
(\mathrm{ArC} 1), 198(\mathrm{Ar}-\mathrm{CO})\end{array}$ \\
\hline IIIa & $\begin{array}{l}21.93\left(\mathrm{CH}-\left(\mathrm{CH}_{3}\right)_{2}\right), 28.22\left(\mathrm{CO}-\mathrm{CH}_{3}\right), 29.31\left(\underline{\mathrm{CH}}-\left(\mathrm{CH}_{3}\right)_{2}\right), 52.71\left(\mathrm{CH}_{2}-\mathrm{NH}-\right), \\
57.79\left(\underline{\mathrm{CH}}_{2}-\mathrm{CH}-\left(\mathrm{CH}_{3}\right)_{2}\right), 68.1\left(\mathrm{ArO}-\underline{\mathrm{C}} \mathrm{H}_{2}-\right), 72.58(\underline{\mathrm{CH}}-\mathrm{OH}), 116.47(\mathrm{ArC} 2), \\
123.54(\mathrm{ArC} 4), 125.22(\mathrm{ArC} 5), 133.12(\mathrm{ArC}), 138.04\left(\mathrm{CH}=\mathrm{CH}_{\text {fumar }}\right), 140.85 \\
(\mathrm{ArC} 3), 161.03(\mathrm{ArC} 1), 176.52\left(\mathrm{COO}_{\text {fumar }}\right), 206.36(\mathrm{Ar}-\mathrm{CO})\end{array}$ \\
\hline IV & $23.65\left(\mathrm{C}_{\mathrm{pyr}}^{3,4}\right), 26.76\left(\mathrm{CO}-\underline{\mathrm{CH}}_{3}\right), 54.2\left(\underline{\mathrm{CH}}_{2}-\mathrm{N}_{\mathrm{pyr}}\right), 58.34\left(\mathrm{C}_{\mathrm{pyr}}^{2,5}\right), 67.23(\underline{\mathrm{C}} \mathrm{H}-$ \\
\hline
\end{tabular}




\begin{tabular}{|c|c|}
\hline & $\begin{array}{l}\mathrm{OH}), 70.67\left(\mathrm{ArO}-\mathrm{CH}_{2}^{-}\right), 113.26(\mathrm{ArC} 2), 121.31(\mathrm{ArC} 4), 129.58(\mathrm{ArC} 6) \\
129.77 \text { (ArC5), } 138.41 \text { (ArC3), 158.98 (ArC1), } 197.93 \text { (Ar-CO) }\end{array}$ \\
\hline VI & $\begin{array}{l}26.77\left(\mathrm{C}_{\mathrm{pha}}{ }^{4,5}\right), 26.92\left(\mathrm{CO}-\mathrm{CH}_{3}\right), 28.50\left(\mathrm{C}_{\mathrm{pha}}{ }^{3,6}\right), 55.82\left(\mathrm{C}_{\mathrm{pha}}{ }^{2,7}\right), 60.16\left(\mathrm{CH}_{2^{-}}\right. \\
\mathrm{NH}-), 65.89(\underline{\mathrm{CH}}-\mathrm{OH}), 70.58\left(\mathrm{ArO}-\underline{\mathrm{CH}}_{2^{-}}\right), 113.27(\mathrm{ArC} 2), 120.04(\mathrm{ArC} 4), \\
121.26 \text { (ArC6), } 129.57 \text { (ArC5), 138.41 (ArC3), 159.08 (ArC1), 197.94 (Ar- } \\
\mathrm{CO})\end{array}$ \\
\hline Via & 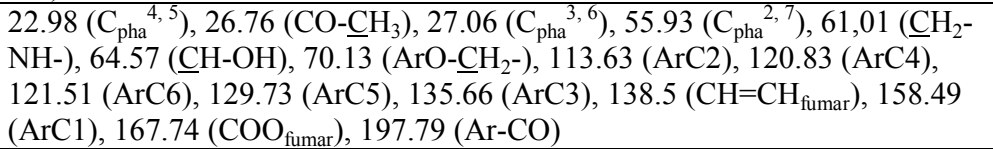 \\
\hline VII & $\begin{array}{l}26.74\left(\mathrm{CO}-\mathrm{CH}_{3}\right), 53.72\left(\mathrm{CH}_{2}-\mathrm{N}_{\text {morf }}\right), 60.88\left(\mathrm{C}_{\text {morf }}^{2,6}\right), 65.31(\mathrm{CH}-\mathrm{OH}), 66.94 \\
\left(\mathrm{C}_{\text {morf }}, 5\right), 70.37\left(\mathrm{ArO}-\mathrm{CH}_{2}-\right), 113.1(\mathrm{ArC} 2), 120.03(\mathrm{ArC} 4), 121.44(\mathrm{ArC} 6), \\
129.6(\mathrm{ArC} 5), 138.42(\mathrm{ArC} 3), 158.89(\mathrm{ArC} 1), 197.85(\mathrm{Ar}-\mathrm{CO})\end{array}$ \\
\hline VIIIa & $\begin{array}{l}29.32\left(\mathrm{CO}-\mathrm{CH}_{3}\right), 45.90\left(\mathrm{~N}_{4 \text { pip }}-{ }^{-} \mathrm{CH}_{3}\right), 52.99\left(\mathrm{C}_{\text {pip }}{ }^{2,6}\right), 55.65\left(\mathrm{C}_{\mathrm{pip}}{ }^{3,5}\right), 61.76 \\
\left(\underline{\mathrm{C}} \mathrm{H}_{2}-\mathrm{N}_{\text {pip }}\right), 69.1\left(\mathrm{ArO}-\underline{\mathrm{CH}}_{2}-\right), 73.32(\underline{\mathrm{C}} \mathrm{H}-\mathrm{OH}), 116.54(\mathrm{ArC} 2), 123.64(\mathrm{ArC} 4), \\
125.06(\mathrm{ArC} 5), 133.08(\mathrm{ArC}), 138.06\left(\mathrm{CH}=\mathrm{CH}_{\text {fumar }}\right), 140.87(\mathrm{ArC} 3), 161.27 \\
(\mathrm{ArC} 1), 176.72\left(\mathrm{COO}_{\text {fumar }}\right), 221.5(\mathrm{Ar}-\mathrm{CO})\end{array}$ \\
\hline
\end{tabular}

$\delta$ chemical schift

Native teicoplanin contains in its structure carbohydrate moieties with functional groups that permit hydrogen and $\pi-\pi$ bonds, electrostatic interactions as well as hydrogen and steric repulsion hindrances.

In the mobile phase methanol/acetonitrile/acetic acid/trietylamine (45/55/0.3/0.2 $\mathrm{v} / \mathrm{v} / \mathrm{v} / \mathrm{v})$, the amount of the acid is relatively higher to the amount of base. Therefore, the ionisation of analytes is assured and ionic interactions of the stationary phase with functional groups are also probable. The presence of methanol in the mobile phase supports the production of hydrogen bonds, which have an effect on the resolution of enantiomers. The results of the enantioseparation are summarized in Table 6 . It is evident that the character of substituent on the basic nitrogen has influence on enantioseparation of the prepared racemic compounds.

Table 6. Chromatographic data for the enantioseparation on teicoplanin bonded chiral stationary phase (Chirobiotic $\mathbf{T})$

\begin{tabular}{|l|l|l|l|l|}
\hline Compound & $\mathrm{t}_{1}$ & $\mathrm{k}_{1}$ & $\alpha$ & $\mathrm{R}_{\mathrm{s}}$ \\
\hline Ia & 18.58 & 3.67 & 1.20 & 2.01 \\
\hline IIa & 16.84 & 3.23 & 1.20 & 2.62 \\
\hline IIIa & 16.14 & 3.05 & 1.08 & 1.11 \\
\hline IV & 6.87 & 0.73 & 1.38 & 1.23 \\
\hline VI & 6.60 & 0.66 & - & - \\
\hline VIIa & 5.10 & 0.28 & 1.17 & 0.50 \\
\hline VIIIa & 4.13 & 0.04 & & \\
\hline & 6.45 & 1.62 & & \\
\hline
\end{tabular}

$\mathrm{R}_{\mathrm{s}}$ stereochemical resolution factor, $\mathrm{t}_{1}$ elution time for enantiomer $1, \mathrm{k}_{1}$ retention factor for enantiomer 1, $\alpha$ separation 
Mobile phase: methanol/acetonitrile/acetic acid/trietylamine (45/55/0,3/0,2 v/v/v/v)

The results of the resolution showed that compounds with alkyl substituent (I-III) (isopropyl, tert-butyl, isobutyl) were effectively separated with resolution factors in the range 1.11-2.62 and selectivity factor in 1.08-1.38. Racemic compounds with heterocycles (V-IX) (morpholino, piperidino, azepan-1-yl, 4-methyl piperazine and 4-(2-methoxyphenyl)piperazine-1-yl) showed either any or poor resolution.

The second direct HPLC method was performed using polysaccharide derivatives as the chiral stationary phase. Amylose tris (3,5-dimethylphenylcarbamate) was used in our research, in which 3 hydroxy groups of amylose are substituted with carbamate moiety. The mechanism of chiral separation on the amylose tris $(3,5-$ dimethylphenylcarbamate) is thought to involve the formation of complexes between the enantiomeric analytes and chiral cavities in the higher order structures of chiral stationary phase.

The initial analyte-phase interaction begin via hydrogen bond formation with the amide $\mathrm{N}-\mathrm{H}$ and carbonyl groups of the carbamate moiety, followed by $\pi-\pi$ and/or dipoledipole interactions and formation of analyte-phase complexes without the structure of chiral stationary phase.

Mobile phase hexane/ethanol/methanol/diethylamine (85/3.75/11.25/0.1 v/v/v/v) was used for the enantioseparation. The presence of the alcohols had influence on the interactions and resolution by alteration of the steric environment of the chiral cavities. The addition of low concentration of the basic additive diethylamine improved the chromatography via interaction with chiral stationary phase.

The influence of the structure of alcohol modifier and its content in mobile phase was studied in the work of Valentova, (2003). From Table 7 and Figs. 1 - 4 it is evident that effective enantioseparation was achieved for all the prepared compounds with resolution factor 2.20-21.80 and selectivity factors 1.17-4.25.

Table 7. Chromatographic data for enantioseparation of prepared compounds on amylose tris(3,5-dimethylphenylcarbamate) bonded chiral stationary phase (Chiralpak AD)

\begin{tabular}{|l|l|l|l|l|}
\hline Compounds & $\mathrm{t}_{1}$ & $\mathrm{k}_{1}$ & $\alpha$ & $\mathrm{R}_{\mathrm{s}}$ \\
\hline $\mathrm{I}$ & 11.42 & 1.99 & 2.84 & 19.35 \\
\hline II & 9.10 & 1.39 & 4.25 & 21.80 \\
\hline IIIa & 16.45 & 3.33 & 2.18 & 17.12 \\
\hline IV & 23.17 & 5.10 & 1.40 & 7.66 \\
\hline IV $*$ & 8,78 & 1.42 & 1.50 & 2.97 \\
\hline V & 31.48 & 5.52 & 1.17 & 2.20 \\
\hline V* & 34.93 & 4.72 & 1.16 & 3.34 \\
\hline VI & 33.46 & 7.78 & 1.29 & 6.03 \\
\hline VII & 46.84 & 11.26 & 1.38 & 9.70 \\
\hline VIII & 29.14 & 6.65 & 1.68 & 10.78 \\
\hline
\end{tabular}

*Mobile phase: hexane/ethanol/methanol/dietylamine $(80 / 10 / 10 / 0.1 \mathrm{v} / \mathrm{v} / \mathrm{v} / \mathrm{v})$

$\mathrm{R}_{\mathrm{s}}$ stereochemical resolution factor, $\mathrm{t}_{1}$ elution time for enantiomer $1, \mathrm{k}_{1}$ retention factor for enantiomer 1, $\alpha$ separation factor 


\section{CONCLUSION}

This study presents synthesis and HPLC enantioseparation of some newly synthesized derivatives of 3-hydroxyphenylethanone of the aryloxyaminopropanol type. The series of compounds were prepared by a well-tried method. Enantioseparation was performed by using two chiral stationary phases. Chiralpak AD was more suitable for enantioseparation of these types of compounds, especially with heterocycles in the basic part of a molecule, rather than antibiotic type teicoplanin and vancomycin of chiral stationary phase.

\section{REFERENCES}

Abe I, Nishiyama T, Nakahara T, Frank H. Gas chromatographic enantiomer separation of pharmaceuticals on capillary columns coated with novel chiral polysiloxanes. J Chromatogr A. 1995;694:237-243.

Aboul-Enein HY, Bakr SA. Enantiomeric resolution of propranolol and analogs on two cellulose (Chiralcel of and OC) and one amylose (Chiralpak Ad) chiral stationary phases. J Liq Chrom Relat Tech. 1998;8:1137-1145.

Beck GM, Neau SH. Optimization of lambda-carrageenan as a chiral selector in capillary electrophoresis separations. Chirality. 2000;12:614-20.

Bhushan R, Arora M. Direct enantiomeric resolution of $( \pm)$ atenolol, $( \pm)$ metoprolol, and $( \pm)$ propranolol by impregnated TLC using L-aspartic acid as chiral selector. Biomed Chrom. 2003;17:226-230.

Bhushan R, Tanwar S. Direct TLC resolution of atenolol and propranolol into their enantiomers using different chiral selectors as impregnating reagents. Biomed Chrom. 2008;22:1028-1034.

Bruchatá K, Čižmáriková R, Polakovičová M, Hroboňová K, Pastírová Z. Synthesis, conformational study and HPLC enantioseparation of nitrophenoxyaminopropanols. Acta Facult Pharm Univ Comeniaenae. 2010;57:24-32.

Čižmáriková R, Bruchatá K, Pastírová Z, Lehotay J, Hroboňová K. HPLC and TLC enantioseparation of the nitro-positioned aryloxysubstituted aminopropanols. Pharmazie. 2010;65:387-388.

Čižmáriková R, Račanská E, Hroboňová $\mathrm{K}$, Lehotay J, Ághová Z, Halešová D. Synthesis, pharmacological activity and chromatographic separation of some novel potential $\beta$-blockers of the aryloxyaminopropanol type. Pharmazie. 2003;58:237-241. 
Donnecke J, Paul C, König WA, Svensson LA, Gyllenhaal O, Vessman J. Immobilization of heptakis 6-O-terc-butyldimethylsilyl-2,3-O-methyl)- $\beta$-cyclodextrin for capillary gas chromatography and SFC and micro-liquid chromatography. J Microcolumn Sep. 1996;8:495-505.

Gyllenhaal O, König WA, Vessman J. Enantiomer separation of metoprolol and its analogues and metabolites by capillary column gas chromatography after derivatization with phosgene. J Chromatogr A. 1985;350:328-331.

Haginaka J, Okazaki Y, Matsunaga H. Separation of enantiomers on a chiral stationary phase based on ovoglycoprotein. V. Influence of immobilization method on chiral resolution. J Chromatogr A. 1999;840:171-181.

Henriksson H, Petterson G, Johansson G. Discrimination between enantioselective and non-selective binding sites on cellobiohydrolase-based stationary phases by site specific competing ligands. J Chromatogr A. 1999;857:107-115.

Hroboňová K, Lehotay J, Čižmáriková R. HPLC enantioseparation of potential $\beta$ blockers of the aryloxyaminopropanol type. Pharmazie. 2004;59:828-832.

Hroboňová K, Lehotay J, Čižmáriková R. HPLC separation of enantiomers of some potential $\beta$-blockers of the aryloxyaminopropanol type using macrocyclic antibiotic chiral stationary phases. Pharmazie. 2005;60:888-891.

Juvancz Z, Grolimund K, Schurig V. Pharmaceutical application of a bonded cyclodextrin stationary phase. J Microcolumn Sep. 1993;5:459-468.

Makamba H, Andrisano V, Gotti R, Cavrini V, Felix G. Sparteine as mobile phase modifier in the chiral separation of hydrophobic basic drugs on an $\alpha_{1}$-acid glycoprotein column. J Chromatogr A. 1998;818:43-52.

Matchett MW, Branch SK, Jefferies TM. Polar organic chiral separation of propranolol and analogues using a $\beta$-cyclodextrin bonded stationary phase. Chirality. 1996;8:126130

Park KL, Kim KH, Jung SH, Lim HM, Hong CH, Kang JS. Enantioselective stabilization of inclusion complexes of metoprolol in carboxymethylated $\beta$-cyclodextrin. J Pharmaceut Biomed Anal. 2000;27:569-576.

Petersen PV, Ekelund J, Olsen L, Ovesen SV. Chiral separations of $\beta$-blocking drug substances using Pirkle-type $\alpha$-Burke 1 chiral stationary phase. J Chromatogr A. 1997;757:65-71.

Proksa B. Capillary zone electrophoretic separation of (R,S)-metipranolol and related substances. Pharmazie. 1999;54:431-434. 
Proksa B, Čižmáriková R. Separation of $\beta$-adrenolytics derivated from 4hydroxyacetophenone by capillary electrophoresis in the presence of cyclodextrins. Anal Chim Acta. 2001;434:75-79.

Rastogi SN, Anand N, Gupta PP, Sharma JN. Agents acting on the central nervous system. 19. ( \pm -1-(o- and m-Alkanoylphenoxy)-3-( $\mathrm{N}^{4}$-arylpiperazinyl)propan-2-ols as local anesthetics, hypotensives, and tranquilizers. J Med Chem. 1973;16:797-804.

Sharma SC, Evans MB, Evans SJ. The enantiomeric separation of metipranolol and desacetylmetipranolol on a cellulose tris-3,5-dimethylphenylcarbamate chiral stationary phase. J Pharm Biomed Anal. 1995;13:129-137.

Valentová J, Čižmáriková R, Bui TTT, Drake AF, Hutt AJ. Enantiomeric resolution of novel aryloxyaminopropanol derivatives with $\beta$-adrenoceptor antagonist activity on a derivatised amylose chiral stationary phase. Chromatographia. 2003;58:733-740.

Zhang H, Shao H, Youmei A, Zhang Z. Optimized conditions of enantioseparation of $\beta$ blockers by CZE using carboxymethyl $\beta$-cyclodextrin as chiral selector. Chromatographia. 2008;68:653-658.

\section{ACKNOWLEDGMENTS}

This publication is the result of the project implementation: Centrum excelentnosti bezpečnostného výskumu kód ITMS: 26240120034 supported by the Research \& Development Operational Programme funded by the ERDF (grant FaF UK/16/2012), and the Scientific grant agency of the Ministry of Education of the Slovak Republic and of Slovak Academy of Sciences (grant no. 1/0164/11).

The Authors wish to thank Dr. Karlovska for recording the NMR and Mrs. Nedorostova for IR and UV spectra and Mr. Lehotsky for recording the HPLC.

Registered: October 4, 2012

Accepted: November, 15, 2012
Doc. RNDr. Ružena Čižmáriková PhD.

Comenius University in Bratislava

Faculty of Pharmacy

Odbojárov 10

83232 Bratislava

Slovak Republic

cizmarikova@fpharm.uniba.sk 


\section{SYNTÉZA A HPLC-ENANTIOSEPARÁCIA DERIVÁTOV 3-HYDROXYFENYLETANÓNU}

${ }^{1}$ Čižmáriková, R. $-{ }^{1}$ Némethy, A. $-{ }^{1}$ Valentová, J. $-{ }^{2}$ Hroboňová, K. $-{ }^{1}$ Bruchatá, K.

${ }^{1}$ Univerzita Komenského v Bratislave, Farmaceutická fakulta, Katedra chemickej teórie liečiv

${ }^{2}$ Slovenská technická univerzita v Bratislave, Fakulta chemickej a potravinárskej technológie, Ústav analytickej chémie

V rámci štúdia syntézy a HPLC enantioseparácie bola osvedčenou metódou pripravená séria 9 derivátov 3-hydroxyfenyletanónu aminopropanolového typu s rozvetvenými alkylmi a heterocyklami v bázickej časti molekuly. Štruktúra pripravených zlúčenín bola potvrdená interpretáciou IR, UV, ${ }^{1} \mathrm{H}$ NMR a ${ }^{13} \mathrm{C}$ NMR spektier. Enantioseparácia bola uskutočnená HPLC technikou na teikoplanínovej (Chirobiotic T) a na amylóze tris (3,5-dimetylfenylkarbamátovej) (Chiralpak AD) chirálnej stationárnej fáze. Porovnaním získaných výsledkov sa potvrdilo, že chirálna stationárna fáza založená na derivatizovanej amylóze je vhodnejšia na enantioseparačné delenie pripravených zlúčenín, hlavne s heterocyklom v bázickej časti molekuly.

Acta Fac. Pharm. Univ. Comen. LIX, 2012, (2), p. 15-27. 